\title{
DESENVOLVIMENTO E VALIDAÇÃO DE METODOLOGIA ANALÍTICA POR CLAE-IR PARA DETERMINAÇÃO DE ARTEMISININA EM Artemisia annua $\mathrm{L}$.
}

\author{
Renata Maria dos Santos Celeghini* \\ Departamento de Tecnologia de Alimentos, Faculdade de Engenharia de Alimentos, Universidade Estadual de Campinas, \\ CP 6121, 13083-862 Campinas - SP, Brasil \\ Ilza Maria de Oliveira Sousa, Ana Paula da Silva, Rodney Alexandre Ferreira Rodrigues e Mary Ann Foglio \\ Centro Pluridisciplinar de Pesquisas Químicas, Biológicas e Agrícolas, Universidade Estadual de Campinas, CP 6171, \\ 13081-970 Campinas - SP, Brasil
}

Recebido em 19/3/08; aceito em 24/11/08; publicado na web em 6/3/09

\begin{abstract}
DEVELOPMENT AND VALIDATION OF ANALYTICAL METHODOLOGY BY HPLC-IR FOR EVALUATION OF ARTEMISININ ON Artemisa aпnиa L. The aim of this work was to develop and validate an analytical methodology for determination of artemisinin used as antimalaric. The method was based on high performace liquid chromatography, using a CN column with mobile phase composed of methanol : $\mathrm{H}_{2} \mathrm{O} 50: 50(\mathrm{~V} / \mathrm{V})$. The results showed that the method presented linearity from 50 to $1500 \mu \mathrm{g} / \mathrm{mL}$. It was considered selective, accurate, precise according to the specific resolution from ANVISA, the Brazilian regulatory agency.
\end{abstract}

Keywords: Artemisia annua L.; clean-up; liquid chromatographic.

\section{INTRODUÇÃO}

A Artemisia annua L. (Asteraceae) é originária de regiões de clima temperado da Ásia e tem sido utilizada pela medicina tradicional chinesa há vários séculos no tratamento de malária. Pesquisadores chineses estudaram mais de 30 espécies do gênero Artemisia para confirmar a atividade antimalárica das mesmas. Somente $A$. annua e A. apiacea Hance foram eficazes em combater o Plasmodium vivax e o Plasmodium falciparum, agentes etimológicos da malária. ${ }^{1}$ Entre os metabólitos secundários isolados das diversas espécies do gênero Artemisia os mais característicos são as lactonas sesquiterpênicas. ${ }^{2}$

A artemisinina (qinghaosu) (Figura 1), lactona sesquiterpênica, apresenta um grupo endoperóxido na molécula, que demonstrou ser essencial para a atividade esquizonticida e possui fundamental importância no tratamento de formas de malária resistente à terapia convencional com drogas, como cloroquina e mefloquina. ${ }^{3} \mathrm{~A}$ partir da artemisinina, já foram obtidos outros derivados semi-sintéticos com maior potência antimalárica, como o artesunato e o arteméter. ${ }^{4}$

Cerca de $40 \%$ da população mundial apresenta risco significativo de contrair a malária. Anualmente, cerca de 250 milhões de pessoas contraem a doença e mais de 1,5 milhões delas, sendo a maioria composta por crianças africanas, morrem vítimas desta doença. Em pacientes com malária grave e complicada, a mortalidade atinge entre 20 e 50\% dos casos. ${ }^{5}$ No Brasil, de acordo com o Anuário Estatístico de Saúde, editado pelo Ministério da Saúde, dentre as doenças de notificação compulsória, a malária atingiu em 1998, 1999 e 2000, valores de incidência bastante elevados, com 471.892, $637.472 \mathrm{e}$ 615.245 casos notificados, respectivamente, tornando-se, portanto, aquela de maior incidência, seguida pela dengue. ${ }^{6}$

A malária atualmente ainda atinge mais de 500 milhões de pessoas levando a óbito acima de 1 milhão de vítimas anualmente. ${ }^{7}$ Apesar destes números, Snow et al. $^{8}$ citam que o número de casos é $50 \%$ maior que os dados oficiais divulgados pela OMS.

Considerando que o teor médio de artemisinina da A. annua L., alcançado na espécie híbrida adaptada no CPQBA, ${ }^{9}$ é de aproximadamente

*e-mail: celeghin@fea.unicamp.br
$1,2 \%$ sobre o peso seco da folha, aliado à informação que o gênero Artemisia (Asteraceae) possui lactonas sesquiterpênicas com diversas atividades biológicas, foi estudado o aproveitamento do resíduo de extração da artemisinina. Constatou-se que o resíduo gerado no processo de extração da artemisinina apresentava atividade antiulcerogênica, provavelmente devido à presença de lactonas sesquiterpênicas, deoxiartemisinina e dihidroepideoxiartenuína b (Figura 1), que parecem aumentar o conteúdo de prostaglandinas na mucosa gástrica. ${ }^{10,11}$ Para viabilizar o controle de qualidade do processo descrito acima percebemos a necessidade de uma metodologia de controle de qualidade que permitisse monitorar o princípio ativo antimalárico simultaneamente com as lactonas sesquiterpênicas, deoxiartemisinina e di-hidroepideoxiartenuína b, contidas no extrato bruto. Diante da semelhança da estrutura química dos compostos $\mathbf{1}$ e 2 (Figura 1), com exceção do grupo endoperóxido, propusemos determinar uma metodologia rápida e eficiente capaz de monitorar a artemisinina e no futuro, quando estiverem disponíveis padrões analíticos de alta pureza, as outras duas lactonas presentes na planta.

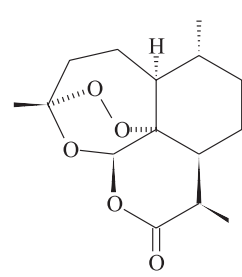

1

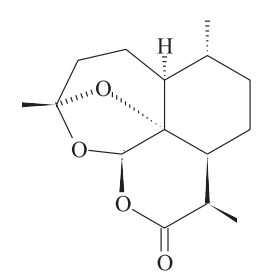

2

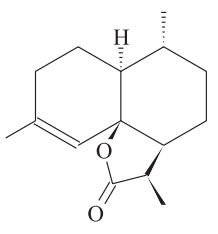

3
Figura 1. Estrutura das lactonas sesquiterpênicas artemisinina 1, deoxiartemisinina $\mathbf{2}$ e di-hidroepideoxiartenuína $b \mathbf{3}$

A cromatografia líquida de alta eficiência CLAE tem sido amplamente empregada para quantificar a artemisinina e os compostos análogos, utilizando-se vários métodos de detecção. Zhao e Zeng ${ }^{12}$ e Qian et al.,$^{13}$ utilizaram a técnica de derivatização pré-coluna para converter a artemisinina em um composto com absorção no UV, aumentando o coeficiente de excitação para comprimentos de onda mais altos. Green et al. ${ }^{14}$ utilizaram o detector de quimioluminescência, 
determinando o limite de detecção de 2,5 ng; entretanto, a resposta do detector diminuiu muito para os derivados da artemisinina.

Vários autores utilizaram o detector eletroquímico para quantificar a artemisinina com boa sensibilidade e limites de detecção entre 1-10 ng. A utilização do detector eletroquímico requer que a detecção do analito ocorra em ausência de oxigênio; para isto, a fase móvel deve ser rigorosamente desoxigenada antes e durante a análise. O instrumento também deve ser mantido em condição completamente livre de oxigênio em todas as linhas e junções. ${ }^{15}$

Avery et al. ${ }^{16}$ analisaram e quantificaram a artemisinina e seus derivados utilizando a cromatografia líquida com detecção de espalhamento de luz, com limite de detecção para os compostos na faixa entre 6 e $60 \mathrm{ng}$.

Maillard et al. ${ }^{17}$ foram os primeiros a analisarem artemisinina em amostras de A. апnиa com cromatografia líquida acoplada ao espectrômetro de massas termospray. Sahai et al. ${ }^{18}$ utilizaram a técnica CLAE-MS com o monitoramento do íon seletivo (SIM) e obtiveram ótimos resultados nas análises de artemisinina. Wang et al..$^{19}$ modificaram a metodologia desenvolvida por Sahai et al. ${ }^{18} \mathrm{e}$ aplicaram nas análises de quantificação de artemisinina em extratos de A. апnua com resultados rápidos e sensíveis.

Outros métodos aplicados no estudo para quantificação da artemisinina foram cromatografia líquida capilar, cromatografia com fluido supercrítico, ${ }^{20}$ cromatografia em camada delgada, ${ }^{21}$ voltametria cíclica $^{22}$ e eletroforese capilar. ${ }^{23}$ A maioria dos artigos contempla análises apenas com padrões e as referências que descrevem a utilização de extratos não levaram em consideração o número de etapas envolvidas no processo de limpeza e a recuperação do analito durante as extrações.

A etapa de limpeza é fundamental para eliminar compostos provenientes da matriz presentes no extrato, que podem interferir no método analítico a ser manipulado. No caso de análises cromatográficas, além de evitar a interferência de contaminantes da matriz que possam coeluir com o analito de interesse, a remoção de interferentes em geral é importante para garantir a longevidade das colunas analíticas. ${ }^{24}$

Rehder et $a .^{25}$ desenvolveram uma metodologia de análise por CLAE com detector de índice de refração para dosagem de artemisinina em folhas de Artemisia annua L., sem considerar a interferência da deoxiartemisinina e di-hidroepideoxiartenuína b. Nestas condições de análise não é possível identificar os interferentes citados acima, pois podem co-eluir com a artemisinina, ocasionando aumento do seu teor.

Em trabalho anterior descrevemos uma metodologia de limpeza e separação de amostras provenientes de folhas secas de $A$. annua. ${ }^{26}$ As condições de análise utilizadas possibilitaram a separação da artemisinina das demais lactonas sesquiterpênicas, contemplando a substituição dos solventes da fase móvel acetonitrila por metanol e substituição do sistema de tampão fosfato por água. ${ }^{25}$

O objetivo deste trabalho foi validar a metodologia analítica para quantificação de artemisinina em extratos de A. annua sem submeter as amostras a modificações químicas, possibilitando estudos posteriores de estabilidade do princípio ativo nos extratos brutos. Optou-se por utilizar o detector de índice de refração por ser universal e possibilitar o monitoramento simultâneo de compostos interferentes como a deoxiartemisinina e di-hidroepideoxiartenuína b, sem necessitar da etapa de derivatização.

Nos últimos anos tem crescido a necessidade de demonstrar a qualidade de medições químicas, através de sua comparabilidade, rastreabilidade e confiabilidade dos dados analíticos, sendo cada vez mais exigida a validação de métodos analíticos. ${ }^{27}$ No Brasil, a ANVISA ${ }^{28}$ (Agência Nacional de Vigilância Sanitária) em 2003, baseada no Q2B da ICH, ${ }^{29}$ publicou a Resolução RE n ${ }^{\circ} 899$ que estabelece parâmetros para validação de métodos bioanalíticos aplicáveis a produtos farmacêuticos. Com o objetivo de assegurar a confiabilidade dos resultados obtidos, validou-se a metodologia analítica de quantificação de artemisinina em amostras da espécie Artemisia annua L.

\section{PARTE EXPERIMENTAL}

\section{Material vegetal}

Foram utilizadas as partes aéreas da espécie vegetal Artemisia annua L. híbrido Ch x Viet 55, cultivada no campo experimental do CPQBA/UNICAMP com voucher Híbrido CPQBA 2/39xPL5. As coletas foram no período em que foi observado o início de florescimento, (aproximadamente de 16-20 semanas após plantio em campo), quando se observou um pico máximo na produção de artemisinina. ${ }^{2}$

O material vegetal foi seco a $40{ }^{\circ} \mathrm{C}$ em estufa com ventilação forçada durante $24 \mathrm{~h}$, moído e armazenado em freezer.

\section{Reagentes}

Os solventes utilizados para as extrações e limpeza das amostras foram grau analítico (Synth, São Paulo, Brasil). Para as análises cromatográficas utilizou-se: padrão de artemisinina (99\%, Sigma, Missouri, USA), solventes grau CLAE (Mallincrodt, Kentucky, USA) e água purificada em sistema Milli-Q (Millipore, São Paulo, Brasil). Para a extração em fase sólida utilizaram-se cartuchos Strata FL-PR Florisil (170 $\mu \mathrm{m}, 80 \mathrm{~A})$ (Phenomenex, California, USA).

\section{Equipamento e condições cromatográficas}

Foi utilizado um cromatógrafo líquido Waters (Massachusetts, USA), bomba Waters 515, válvula injetora Rheodyne, com alça de amostragem de $20 \mu \mathrm{L}$. O sistema foi acoplado a um detector de índice de refração Waters 2414; temperatura interna do detector 35 ${ }^{\circ} \mathrm{C}$; temperatura da coluna $35^{\circ} \mathrm{C}$. Os dados cromatográficos foram analisados usando-se software do equipamento Empower-Waters. Utilizou-se coluna com fase estacionária ciano, Luna CN $5 \mu \mathrm{m} 250$ x 4,6 mm (Phenomenex, California, USA); fase móvel metanol:água $(50: 50 \mathrm{v} / \mathrm{v})$; modo isocrático e vazão $1,0 \mathrm{~mL} / \mathrm{min}$.

\section{Extração}

Os extratos foram obtidos a partir de $0,500 \mathrm{~g}$ de folhas secas e moídas com 3 extrações com $5 \mathrm{~mL}$ de metanol, em Ultra Turrax (Quimis, São Paulo, Brasil), por $3 \mathrm{~min}, 10.000 \mathrm{rpm}$ à temperatura ambiente. $\mathrm{O}$ extrato foi filtrado a vácuo em funil de placa porosa e evaporado à secura sob vácuo em evaporador rotativo (Büchi, Flawil, Switzerland).

\section{EFS-limpeza ${ }^{26}$}

Os cartuchos de Florisil ${ }^{\circledast}$ (Strata 8B-SO13-HCH, Phenomenex, California, USA), foram conectados ao sistema de extração a vácuo (Manifold) (J. T. Baker, New Jersy, USA) condicionados com 10 $\mathrm{mL}$ de hexano; em seguida introduziu-se o extrato dissolvido em $2 \mathrm{~mL}$ de diclorometano, eluindo com $3 \mathrm{~mL}$ de hexano e $60 \mathrm{~mL}$ de diclorometano, coletando-se as frações separadamente. A fração de diclorometano foi evaporada à secura, sob vácuo em evaporador rotativo e ressuspendida em balão volumétrico de $5 \mathrm{~mL}$ com metanol grau CLAE. As amostras foram filtradas (membranas Durepore PVDF $0,45 \mu \mathrm{m}$, Millipore) e analisadas em triplicata por CLAE/IR.

\section{Validação do método}

O método foi validado de acordo com a resolução $R E n^{\circ} 899$, de 29 de maio de 2003, da ANVISA. ${ }^{28}$ 


\section{Verificação de interferentes}

Foi realizado todo o processo de extração e limpeza sem a presença da amostra ou padrão, para se observar a presença de interferentes no tempo de retenção da artemisinina.

\section{Linearidade}

A curva de calibração foi obtida com 5 pontos das soluçõespadrão em metanol nas concentrações de 50 a $1500 \mu \mathrm{g} / \mathrm{mL}$. Os padrões foram preparados em triplicata e injetados em quintuplicata $(n=5)$. A linearidade foi avaliada em dias diferentes e foi estimada pela análise de regressão linear através do método dos mínimos quadrados.

\section{Limite de detecção e de quantificação}

A estimativa do limite de detecção (LD) foi feita com base na relação de 3 vezes o ruído da linha de base, por diluições sucessivas do padrão de artemisinina. O limite de quantificação (LQ) foi determinado através do ruído da linha de base e foi considerado como a concentração que produziu a relação sinal-ruído superior a 10:1. ${ }^{27}$

\section{Recuperação}

A exatidão foi avaliada em termos de recuperação através do método de adição padrão. Este método é usado quando é difícil ou impossível preparar um branco da matriz sem a substância de interesse. ${ }^{28}$ Quantidades conhecidas de artemisinina foram adicionadas em triplicata na planta moída em três níveis de concentração baixo $(250 \mu \mathrm{g} / \mathrm{mL})$, médio $(650 \mu \mathrm{g} / \mathrm{mL})$ e alto $(1050 \mu \mathrm{g} / \mathrm{mL})$, em seguida extraídas conforme o procedimento de preparo da amostra. A amostra sem adição de padrão e cada uma das amostras com o padrão adicionado (concentração estimada) foram analisadas e a recuperação foi expressa em termos de porcentagem da quantidade medida da substância em relação à quantidade adicionada na matriz.

\section{Precisão}

A precisão do método foi avaliada levando em consideração a repetibilidade e a precisão intermediária. Para avaliar a repetibilidade analítica da metodologia empregada na análise, prepararam-se 6 amostras que foram injetadas em triplicata no mesmo dia, nas mesmas condições cromatográficas e pelo mesmo analista. As respostas foram expressas como o coeficiente de variação. No caso da precisão intermediária foram preparadas 6 amostras, avaliadas em 3 dias diferentes por analistas diferentes. As amostras foram injetadas em triplicata e os resultados expressos como coeficiente de variação. Os resultados foram avaliados e checados pelos critérios do teste de Cochran. ${ }^{30}$

\section{RESULTADOS E DISCUSSÃO}

As curvas analíticas traçadas por padronização externa para artemisinina demonstraram excelentes linearidade na faixa linear de $50-1500 \mu \mathrm{g} / \mathrm{mL}$ com coeficiente de correlação médio de 0,999 . Isto indica a validade do método, pois obedece a uma correlação linear nos intervalos de concentração avaliados. A curva de calibração obtida pelo método dos mínimos quadrados pode ser expressa pela equação $\mathrm{y}=1326,505 \mathrm{x}+54048,021$.

O limite de detecção (LD) obtido neste trabalho foi de $2,2 \mu \mathrm{g} /$ $\mathrm{mL}$ e o limite de quantificação (LQ) foi considerado como 10 vezes a relação sinal ruído, portanto, 7,5 $\mu \mathrm{g} / \mathrm{mL}$. Esses resultados demonstram que o método é sensível para detectar e quantificar artemisinina em amostras de Artemisia annua $\mathrm{L}$.

A exatidão do método foi determinada por meio da obtenção da porcentagem de recuperação média avaliada em três níveis de concentrações em triplicata. Os valores obtidos encontram-se descritos na Tabela 1; a amostra sem adição de padrão apresentou concentração de artemisinina de $350 \mu \mathrm{g} / \mathrm{mL}$, portanto, as amostras adicionadas apresentaram concentrações estimadas de 600,1000 e $1400 \mu \mathrm{g} / \mathrm{mL}$.

A determinação da recuperação do analito foi realizada através da sua quantificação na matriz em estudo pelo emprego de um padrão analítico. O grau de recuperação variou entre 102,7 e 103,9\%, o que está dentro das especificações esperadas para matrizes complexas $(80-120 \%){ }^{28}$

Tabela 1. Porcentagem de recuperação de artemisinina no processo de extração e limpeza em três níveis de concentração

\begin{tabular}{lccc}
\hline $\begin{array}{l}\text { Concentração } \\
\text { estimada } \\
(\mu \mathrm{g} / \mathrm{mL})\end{array}$ & $\begin{array}{c}\text { Média concentração } \\
\text { Experimental CLAE } \\
(\mu \mathrm{g} / \mathrm{mL})\end{array}$ & $\begin{array}{c}\text { Recuperação } \\
\text { clean-up } \\
(\%)\end{array}$ & $\begin{array}{c}\mathrm{CV} \\
(\%)\end{array}$ \\
\hline 600,00 & 623,40 & 103,90 & 1,21 \\
1000,00 & 10029,00 & 102,90 & 1,68 \\
1400,00 & 1437,80 & 102,70 & 1,57 \\
\hline
\end{tabular}

CV- coeficiente de variação; $n=6$

A precisão do método (Tabela 2) foi avaliada através da repetibilidade envolvendo várias medições realizadas no mesmo dia, nas mesmas condições cromatográficas e pelo mesmo analista. A precisão intermediária foi avaliada por analistas diferentes em 3 dias diferentes. Os resultados da repetibilidade e precisão intermediária encontram-se na Tabela 2, como média das concentrações e dos desvios padrões encontrados e expressos como coeficiente de variação.

Tabela 2. Repetibilidade e precisão intermediária do método cromatográfico para determinação de artemisinina

\begin{tabular}{lcc}
\hline Analista & $\mathrm{A}$ & $\mathrm{B}$ \\
\hline Repetibilidade $^{\mathrm{a}}$ & $\begin{array}{c}\text { Precisão }^{\text {intermediária }} \\
\text { Média (\% p/p) }\end{array}$ & 0,849 \\
Desvio padrão & 0,829 & 0,006 \\
DPR & 0,005 & 0,007 \\
CV $(\%)$ & 0,007 & 0,656 \\
\hline
\end{tabular}

$\mathrm{CV}$ - coeficiente de variação; $\mathrm{a}=$ mesmo dia, mesmo analista e mesmo equipamento; $b=$ três dias diferentes, analistas diferentes e mesmo equipamento.

Os valores do coeficiente de variação demonstrados na Tabela 2 confirmam que os resultados estão dentro dos critérios aceitáveis $(\mathrm{CV} \leq 5 \%)$. Segundo a ANVISA, ${ }^{28}$ o valor máximo aceitável deve ser definido de acordo com a metodologia empregada, concentração do analito na amostra, tipo da matriz, não se admitindo valores superiores a $5 \%$.

A variância entre os dois analistas foi verificada através da aplicação do método de Cochran, ${ }^{30}$ apresentando valor calculado menor que o valor tabelado $\left(\mathrm{Cc}=0,510, \mathrm{C}_{\text {tab } 1 \%}=0,793 \mathrm{e}_{\text {tab.5\% }}=0,707\right.$ para $\left.\mathrm{n}=6\right)$ indicando que não há variação significativa entre os resultados.

O método proposto apresentou boa seletividade, como pode ser observado na Figura 2, pois os compostos interferentes deoxiartemisinina e di-hidroepideoxiartenuína b não coeluem com a artemisinina, demonstrando a especificidade do método em relação ao analito citado. 


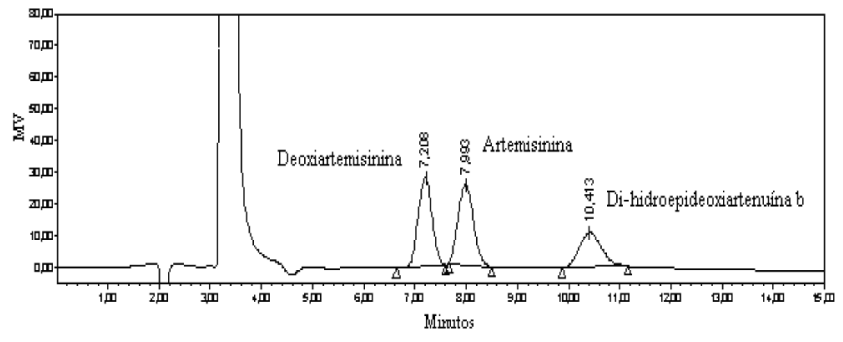

Figura 2. Perfil cromatográfico da separação da artemisinina dos compostos interferentes deoxiartemisinina e di-hidroepideoxiartenuína $b$

\section{CONCLUSÕES}

O método analítico proposto para determinação de artemisinina em amostras de Artemisia annua L., utilizando coluna com fase estacionária ciano e detecção por índice de refração, pode ser considerado como método alternativo, seletivo, exato, preciso, rápido e econômico quando comparado com a metodologia descrita por Qian et al..$^{13}$ que utiliza detector UV e requer a etapa da derivatização para quantificação da artemisinina. A metodologia desenvolvida mostrou-se reprodutível e linear na faixa de concentração de 50 a $1500 \mu \mathrm{g} / \mathrm{mL}$, sendo adequada à avaliação da qualidade de amostras que contêm artemisinina.

Os resultados deste estudo permitirão um melhor acompanhamento da extração em planta piloto, possibilitando a quantificação simultânea da artemisinina, deoxiartemisinina e di-hidroepideoxiartenuína b em extratos brutos de Artemisia annua L. e também poderão auxiliar no desenvolvimento do estudo de estabilidade destes extratos.

\section{AGRADECIMENTOS}

À FAPESP (Fundação de Amparo à Pesquisa do Estado de São Paulo), financiadora da bolsa de Pós-doutorado (01/09660-6) e do projeto Auxílio à Pesquisa (2002/03004-2).

\section{REFERÊNCIAS}

1. Dias, C. P.; Dissertação de Mestrado, Universidade Estadual de Campinas, Brasil, 1997.

2. Rodrigues, R. A. F.; Foglio, M. A.; Boaventura Jr. S; Santos, A. S.; Rehder, V. L. G.; Quim. Nova 2006, 29, 368.

3. Meshnick, S. R.; Yang, Y.; Lima, V.; Keiypers, F.; Kamchon, W. S.; Yuthavong, Y.; Antimicrob. Agents Chemother. 1993, 37, 1108.

4. Borstnik, K.; Paik, I.; Shapiro, T.; Posner, G. H.; Int. J. Parasitol. 2002, 32, 1661.

5. Balint, G. A.; Pharmacol. Ther. 2001, 90, 261.

6. http://portal.saude.gov.br/portal/aplicacoes/anuario2001/index.cfm, acessada em Fevereiro 2007.

7. Jiang, L.; Liu, Q.; Chen, P.; Sci. China, Ser. C: Life Sci. 2007, 50, 251.

8. Snow, R. W.; Guerra, C. A.; Noor, A. M.; Myint, H. Y.; Hay, S. I.; Nature 2005, 437, 7056 .
9. Ferreira, J. F. S.; Lauglin, J. C.; Delabays, N.; de Magalhães, P. M.; Plant. Genet. Resour. 2005, 3, 206.

10. Dias, C. P.; Tese de Doutorado, Universidade Estadual de Campinas, Brasil, 2004.

11. Foglio, M. A.; Dias, P. C.; Antonio, M. A.; Possenti, A.; Rodrigues, R. A. F.; Silva, E. F.; Rehder, V. L. G.; Carvalho, J. E.; Planta Med. 2002, 68, 515.

12. Zhao, S. S.; Zeng, M. Y.; Planta Med. 1985, 51, 233.

13. Qian, G.; Yang, Y.; Ren, Q.; J. Liq. Chromatogr. Relat. Technol. 2005, 28, 705 .

14. Green, M. D.; Mount, D. L.; Todd, G. D.; Capomacchia, A. C.; J. Chromatogr., A 1995, 695, 237.

15. Sandrenan, N.; Sioufi, A.; Godbillon, J.; Netter, C.; Donker, M.; vanValkenburg, C.; J. Chromatogr., B: Anal. Technol. Biomed. Life Sci. 1997, 691,145.

16. Avery, B. A.; Venkatesh, K. K.; Avery, M. A.; J. Chromatogr., B: Anal. Technol. Biomed. Life Sci. 1999, 730, 71.

17. Maillard, M. P.; Wolfender, J.L.; Hostettmann, K.; J. Chromatogr. 1993, 647, 147.

18. Sahai, P.; Vishwakarma, R. A.; Bharel, S.; Gulati, A.; Abdin, M. Z.; Srivastava, P. S.; Jain, S. K.; Anal. Chem. 1998, 70, 3084.

19. Wang, M.; Park, C.; Wu, Q.; Simon, J.; J. Agric. Food Chem. 2005, 53, 7010.

20. Kohler, M.; Haerdi, W.; Christen, P.; Veuthey, J.; Methods Biotechnol. 2000, 13, 135.

21. Gupta, M. M.; Jain, D. C.; Verma, R. K.; Gupta, A. P.; J. Med. Aromat. Plant Sci. 1996, 18, 5.

22. Jiang, H. L.; Chen, K. X.; Tang, Y.; Chen, J. Z.; Li, Y.; Wang, Q. M.; Ji, R. Y.; Zhuang, Q. K.; Indian J. Chem., Sect. B: Org. Chem. Incl. Med. Chem. 1997, 36,154.

23. Dhulst, A.; Augustijns, P.; Arens, S.; VanParijs, L.; Colson, S.; Verbeke, N.; Kinget, R.; J. Chromatogr. Sci. 1996, 34, 276.

24. Lanças, F. M.; Extração em fase sólida (SPE), Editora Rima: São Carlos, 2004

25. Rehder, V. L. G.; Rodrigues, M. V. N.; Sartoratto, A.; Foglio, M. A.; Rev. Bras. Farmacogn 2002, 12, 116.

26. Celeghini, R. M. S.; Silva, A. P.; Sousa, I. M. O.; Foglio, M. A.; Rev. Bras. Plantas Med. 2006, 8, 119.

27. Ribani, M.; Bottoli, C. B. G.; Collins, C. H.; Jardim, I. C. S. F.; Quim. Nova 2004, 27, 771.

28. Agência Nacional de Vigilância Sanitária (ANVISA); Guia para Validação de Métodos Analíticos e Bioanáliticos, RE n 899, de 29 de maio de 2003.

29. International Conference on Harmonisation (ICH); Validation of Analytical Procedures: Methodology, Q2B, Step 4, Consensus Guideline, 6 November 1996.

30. International Organization for Standardization, ISO/DIS 5725; Accuracy (trueness and precision) of Measurement Methods and Results. Part 2: A basic method for the determination of repeatability and reproducibility of a standard measurement of method, Geneva: Switzerland, 1990. 